\title{
The existence and approximation of the periodic solutions for system of first order nonlinear differential equationsby using Lebesgue integrable
}

\author{
R.N.Butris \\ Dept. of Mathematics \\ College of Education \\ University of Mosul \\ Received \\ 08/10/2006
}

\author{
Merna Adel Aziz \\ Dept. of Mathematics \\ College of Computer Science \& Math \\ University of Mosul \\ Accepted \\ 09/01/2007
}

\section{المالخص}

يتضمن هذا البمث درلسة وجود وقارب الح لـ ال دوري لظ لمهم ـن المع ادلات

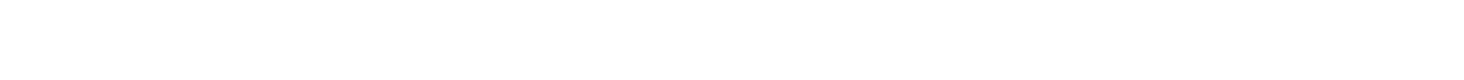

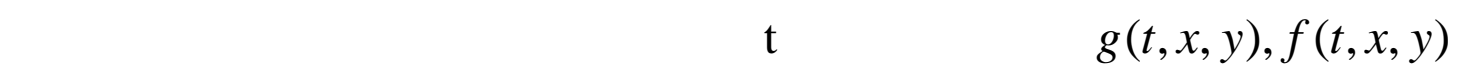

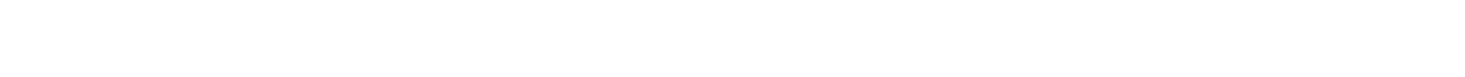
اللإطية لـ ـ ـ Samoilenko.

\section{ABSTRACT}

In this paper we study the existence and approximation of the periodic solutions for a system of first order nonlinear differential equations by assuming that each of the functions $f(t, x, y), g(t, x, y)$ are measurable at $t$ and bounded by Lebesgue integrable functions.

The numerical-analytic method has been used to study the periodic solutions of ordinary differential equations which were introduced by A. M. Samoilenko.

\section{INTRODUCTION}

There are many subjects in physics and technology use mathematical methods that depends on the nonlinear differential equations, and it became clear that the existence of the periodic solutions and its algorithm structure form an important problems in the present time, where many of studies treated autonomous and non autonomous periodic systems and specially with the integral and differential equations and linear and nonlinear integro - differential problems of periodic solutions.

Samoilenko [7] assumed a numerical analytic method to study the periodic solutions for the ordinary differential equations and this method include uniformly sequences of the periodic functions as in the studies $[1,2,3,5,6]$.

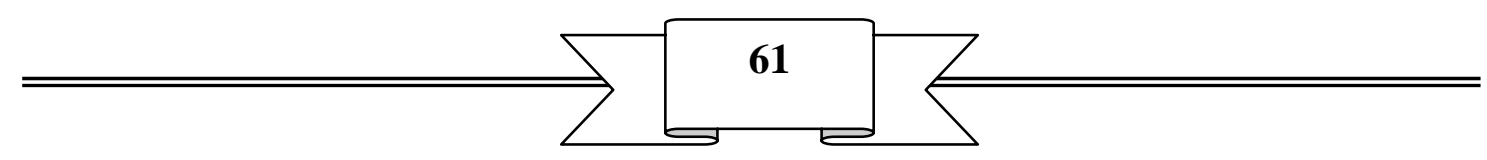


In this paper we consider the system of the non linear differential equations of the form:

$$
\left.\begin{array}{c}
\frac{d x(t)}{d t}=(A+B(t)) x(t)+f(t, x, y) \\
\frac{d y(t)}{d t}=(C+D(t)) y(t)+g(t, x, y)
\end{array}\right\}
$$

where $x \in D \subseteq R^{n}, D$ represents a closed domain and bounded, the each of the functions

$$
\begin{aligned}
& f(t, x, y)=\left(f_{1}(t, x, y), f_{2}(t, x, y), \ldots \ldots . ., f_{n}(t, x, y)\right) \\
& g(t, x, y)=\left(g_{1}(t, x, y), g_{2}(t, x, y), \ldots \ldots ., g_{n}(t, x, y)\right) \\
& \text { are defined, and continuous in the domain }
\end{aligned}
$$$$
(t, x, y) \in[0, T] \times D \times D_{1}
$$

and periodic in $\mathrm{t}$ of period $\mathrm{T}$.

where $y \in D_{1} \subseteq R^{n}, D_{1}$ represents a closed domain and bounded from the Euclidean space $R^{n}$, with the assumption that the two functions $f(t, x, y)$ and $g(t, x, y)$ satisfy the following inequalities:

$$
\begin{aligned}
& \|f(t, x, y)\| \leq m_{1}(t), \quad\|g(t, x, y)\| \leq m_{2}(t) \\
& \left\|f\left(t, x_{1}, y_{1}\right)-f\left(t, x_{2}, y_{2}\right)\right\| \leq K_{1}(t)\left\|x_{1}-x_{2}\right\|+K_{2}(t)\left\|y_{1}-y_{2}\right\| \\
& \left\|g\left(t, x_{1}, y_{1}\right)-g\left(t, x_{2}, y_{22}\right)\right\| \leq L_{1}(t)\left\|x_{1}-x_{2}\right\|+L_{2}(t)\left\|y_{1}-y_{2}\right\|
\end{aligned}
$$

for all $t \in[0, T], x, x_{1}, x_{2} \in D, y, y_{1}, y_{2} \in D_{1}$ where each of $m_{1}(t), m_{2}(t)$, $K_{1}(t), K_{2}(t), L_{1}(t), L_{2}(t)$ are Lebesgue integrable functions in the interval $0 \leq t \leq T$, where $A=\left[A_{i j}\right], B(t)=\left[B_{i j}(t)\right], C=\left[C_{i j}\right], D(t)=\left[D_{i j}(t)\right]$ positive $\mathrm{n} \times \mathrm{n}$ matrices are defined in the domain $-\infty,<0 \leq t \leq T<\infty$ continuous and periodic in $\mathrm{t}$ and satisfy the following inequalities :

$$
\begin{array}{ll}
\left\|e^{A(t-s)}\right\| \leq Q, & \left\|e^{C(t-s)}\right\| \leq R \\
\|B(t)\| \leq H \quad, & \|D(t)\| \leq J \\
\left\|x_{\circ}\right\|=\delta_{\circ} \quad, & \left\|y_{\circ}\right\|=\sigma_{\circ}
\end{array}
$$

Where $-\infty,<0 \leq t \leq T<\infty$ and $\mathrm{Q}, \mathrm{R}, \mathrm{H}, \mathrm{J}, \delta_{\circ}, \sigma_{\circ}$ are positive constants.

Let $0 \leq t \leq b_{1} \leq T$ and $0 \leq t \leq b_{2} \leq T$ where $b_{1}, b_{2}$ are two chosen points so that

$$
\int_{0}^{b_{1}} m_{1}(t) d t \leq c_{1}, \int_{0}^{b_{1}} K_{1}(t) d t \leq c_{2} \leq 1, \int_{0}^{b_{1}} K_{2}(t) d t \leq c_{3} \leq 1
$$




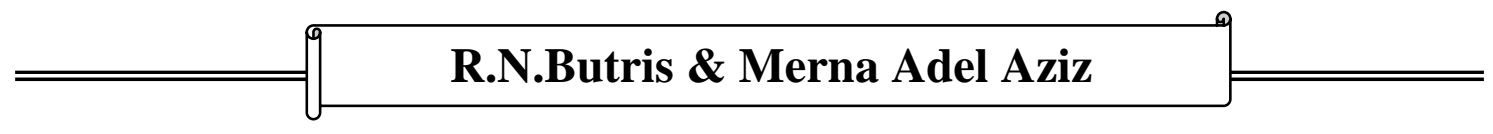

$\int_{0}^{b_{2}} m_{2}(t) d t \leq \delta_{1}, \int_{0}^{b_{2}} L_{1}(t) d t \leq \delta_{2} \leq 1, \int_{0}^{b_{2}} L_{2}(t) d t \leq \delta_{3} \leq 1$

Definition 1 [7] :-

The system of nonlinear differential equations (1) where the righthand side is defined, continuous and periodic in $t$ and has period $\mathrm{T}$ in the domain (2) is said to be system - $\mathrm{T}$ if

1- The two sets $D_{f}, D_{g}$ are not empty

$$
\left.\begin{array}{l}
D_{f}=D-\left(\frac{T}{2} N+Q C_{1}\right) \neq \phi \\
D_{g}=D_{1}-\left(\frac{T}{2} N^{*}+R \delta_{1}\right) \neq \phi
\end{array}\right\}
$$

where $N^{*}=R^{2} J \sigma_{\circ}, N=Q^{2} H \delta_{\circ},\|\|\left\|=\max _{t \in[0 . T]}\right\|_{.}$

2- The greatest eigen value of the matrix

$$
\Omega_{\circ}=\left[\begin{array}{cc}
\frac{T}{2} Q H+Q C_{2} & Q C_{3} \\
R \delta_{2} & \frac{T}{2} R J+R \delta_{3}
\end{array}\right]
$$

is smaller than 1 that is

$$
\lambda_{\text {max }}\left(\Omega_{\circ}\right)=\frac{\rho_{1}+\sqrt{\rho_{1}^{2}-4 \rho_{2}}}{2}<1
$$

Where

$$
\begin{aligned}
& \rho_{1}=\left(\frac{T}{2} Q H+Q C_{2}\right)+\left(\frac{T}{2} R J+R \delta_{3}\right) \\
& \rho_{2}=R \delta_{2} Q C_{3}-\left(\frac{T}{2} Q H+Q C_{2}\right)\left(\frac{T}{2} R J+R \delta_{3}\right)
\end{aligned}
$$

\section{Definition 2 [7]:-}

The value of $\mu^{*}=\left(\mu_{1}^{*}, \mu_{2}^{*}\right)$ at the point $\left(t, x_{\circ}, y_{\circ}\right)$ for the following system:

$$
\left.\begin{array}{l}
\frac{d x(t)}{d t}=(A+B(t)) x(t)+f(t, x, y)-\mu_{1}^{*} \\
\frac{d y(t)}{d t}=(C+D(t)) y(t)+g(t, x, y)-\mu_{2}^{*}
\end{array}\right\}
$$

Is periodic in $\mathrm{t}$ of period $\mathrm{T}$ is called a constant $-\Delta^{*}$ for the system (1) through at the point $t=0, x=x_{\circ}, y=y_{\circ}$ if $\mu^{*}$ is unique at that point. 


\section{Section One : The periodic approximate solution}

Lemma 1 :-

\section{for the system (1)}

Assume that each of $f(t, x, y)$ and $g(t, x, y)$ are vector functions, continuous and defined in the interval $[0, T]$, then the inequality

$$
\left(\begin{array}{l}
\left\|M_{1}\left(t, x_{\circ}, y_{\circ}\right)\right\| \\
\left\|M_{2}\left(t, x_{\circ}, y_{\circ}\right)\right\|
\end{array}\right) \leq\left(\begin{array}{l}
\beta_{1}(t) N+Q C_{1} \\
\beta_{2}(t) N^{*}+R \delta_{1}
\end{array}\right)
$$

holds for $\quad 0 \leq t \leq T, \beta_{1}(t) \leq \frac{T}{2}, \beta_{2}(t) \leq \frac{T}{2}$, where $N^{*}=R^{2} J \sigma_{\circ}, N=Q^{2} H \delta_{\circ}$ $\beta_{1}(t)=\left[\frac{t\left(2 e^{\|A\|(T-t)}-e^{\|A\| T}-\|E\|\right)+T\left(e^{\|A\| T}-e^{\|A\|(T-t)}\right)}{e^{\|A\| T}-\|E\|}\right]$
$\beta_{2}(t)=\left[\frac{t\left(2 e^{\|C\|(T-t)}-e^{\|C\| T}-\|E\|\right)+T\left(e^{\|C\| T}-e^{\|C\|(T-t)}\right)}{e^{\|C\| T}-\|E\|}\right]$, $M_{1}\left(t, x_{\circ}, y_{\circ}\right)=\int_{0}^{t} e^{A(t-s)}\left[B(s) x_{\circ} e^{A t}+f\left(s, x_{\circ}, y_{\circ}\right)-\right.$

$$
\left.-\frac{A}{e^{A T}-E} \int_{0}^{T} e^{A(T-s)}\left[B(s) x_{\circ} e^{A t}+f\left(s, x_{\circ}, y_{\circ}\right)\right] d s\right] d s
$$

$$
\begin{aligned}
M_{2}\left(t, x_{\circ}, y_{\circ}\right)= & \int_{0}^{t} e^{C(t-s)}\left[D(s) y_{\circ} e^{C t}+g\left(s, x_{\circ}, y_{\circ}\right)-\right. \\
& \left.-\frac{C}{e^{C T}-E} \int_{0}^{T} e^{C(T-s)}\left[D(s) y_{\circ} e^{C t}+g\left(s, x_{\circ}, y_{\circ}\right)\right] d s\right] d s
\end{aligned}
$$

Proof :-

$$
\begin{aligned}
& \left\|M_{1}\left(t, x_{\circ}, y_{\circ}\right)\right\| \leq \\
& \leq\left[\|E\|-\left(\frac{e^{\|A\| T}-e^{\|A\|(T-t)}}{e^{\|A\| T}-\|E\|}\right)\right] \int_{0}^{t}\left\|e^{A(t-s)}\right\|\left[\|B(s)\|\left\|x_{\circ}\right\|\left\|e^{A t}\right\|+\left\|f\left(s, x_{\circ}, y_{\circ}\right)\right\|\right] d s+ \\
& \quad+\frac{e^{\|A\| T}-e^{\|A\|(T-t)}}{e^{\|A\| T}-\|E\|} \int_{t}^{T}\left\|e^{A(t-s)}\right\|\left[\|B(s)\|\left\|x_{\circ}\right\|\left\|e^{A t}\right\|+\left\|f\left(s, x_{\circ}, y_{\circ}\right)\right\|\right] d s \\
& =\beta_{1}(t) N+Q C_{1}
\end{aligned}
$$

and also 


$$
\begin{aligned}
& \hline \hline M_{2}\left(t, x_{\circ}, y_{\circ}\right) \| \leq \\
& \quad\left[\|E\|-\left(\frac{e^{\|C\| T}-e^{\|C\|(T-t)}}{e^{\|C\| T}-\|E\|}\right) \int_{0}^{t}\left\|e^{C(t-s)}\right\|\left[\|D(s)\|\left\|y_{\circ}\right\|\left\|e^{C t}\right\|+\left\|g\left(s, x_{\circ}, y_{\circ}\right)\right\|\right] d s+\right. \\
& +\frac{e^{\|C\| T}-e^{\|C\|(T-t)}}{e^{T}\|C\| T}-\|E\| \int_{t}^{C(t-s)} \|\left[\|D(s)\|\left\|y_{\circ}\right\|\left\|e^{C t}\right\|+\left\|g\left(s, x_{\circ}, y_{\circ}\right)\right\|\right] d s \\
& =\beta_{2}(t) N^{*}+R \delta_{1}
\end{aligned}
$$

from (1.2) and (1.3) we conclude that the inequality (1.1) holds for $0 \leq t \leq T, \beta_{1}(t) \leq \frac{T}{2}, \beta_{2}(t) \leq \frac{T}{2}$

\section{Theorem 1:-}

If the system (1) which satisfies the inequalities (3),(4),(5) and the conditions (11), (12) has a periodic solution $x=x\left(t, x_{\circ}, y_{\circ}\right)$, $y=y\left(t, x_{\circ}, y_{\circ}\right)$ passes through the point $\left(t, x_{\circ}, y_{\circ}\right)$, then the sequences of functions:-

$$
\begin{gathered}
x_{m+1}\left(t, x_{\circ}, y_{\circ}\right)=x_{\circ} e^{A t}+\int_{0}^{t} e^{A(t-s)}\left[B(s) x_{m}\left(s, x_{\circ}, y_{\circ}\right)+f\left(s, x_{m}\left(s, x_{\circ}, y_{\circ}\right), y_{m}\left(s, x_{\circ}, y_{\circ}\right)\right)-\right. \\
\left.-\frac{A}{e^{A T}-E} \int_{0}^{T} e^{A(T-s)}\left[B(s) x_{m}\left(s, x_{\circ}, y_{\circ}\right)+f\left(s, x_{m}\left(s, x_{\circ}, y_{\circ}\right), y_{m}\left(s, x_{\circ}, y_{\circ}\right)\right)\right] d s\right] d s
\end{gathered}
$$

with $\quad x_{\circ}\left(t, x_{\circ}, y_{\circ}\right)=x_{\circ} e^{A t} \quad, m=0,1,2, \ldots .$.

$$
\begin{array}{r}
y_{m+1}\left(t, x_{\circ}, y_{\circ}\right)=y_{\circ} e^{C t}+\int_{0}^{t} e^{C(t-s)}\left[D(s) y_{m}\left(s, x_{\circ}, y_{\circ}\right)+g\left(s, x_{m}\left(s, x_{\circ}, y_{\circ}\right), y_{m}\left(s, x_{\circ}, y_{\circ}\right)\right)-\right. \\
\left.-\frac{C}{e^{C T}-E} \int_{0}^{T} e^{C(T-s)}\left[D(s) y_{m}\left(s, x_{\circ}, y_{\circ}\right)+g\left(s, x_{m}\left(s, x_{\circ}, y_{\circ}\right), y_{m}\left(s, x_{\circ}, y_{\circ}\right)\right)\right] d s\right] d s
\end{array}
$$

with $y_{\circ}\left(t, x_{\circ}, y_{\circ}\right)=y_{\circ} e^{C t} \quad, m=0,1,2, \ldots \ldots \ldots$

are periodic in $\mathrm{t}$ of period $\mathrm{T}$ and uniformly convergent as $m \rightarrow \infty$ in the domain

$\left(t, x_{\circ}, y_{\circ}\right) \in[0, T] \times D_{f} \times D_{g}$ 
to the limit functions $x^{\circ}\left(t, x_{\circ}, y_{\circ}\right)$ and $y^{\circ}\left(t, x_{\circ}, y_{\circ}\right)$ which are defined, continuous and periodic in $t$ of period $\mathrm{T}$ in the domain (1.6) satisfy the system of integral equations

$$
\begin{gathered}
x\left(t, x_{\circ}, y_{\circ}\right)=x_{\circ} e^{A t}+\int_{0}^{t} e^{A(t-s)}\left[B(s) x\left(s, x_{\circ}, y_{\circ}\right)+f\left(s, x\left(s, x_{\circ}, y_{\circ}\right), y\left(s, x_{\circ}, y_{\circ}\right)\right)-\right. \\
\left.-\frac{A}{e^{A T}-E} \int_{0}^{T} e^{A(T-s)}\left[B(s) x\left(s, x_{\circ}, y_{\circ}\right)+f\left(s, x\left(s, x_{\circ}, y_{\circ}\right), y\left(s, x_{\circ}, y_{\circ}\right)\right)\right] d s\right] d s \\
y\left(t, x_{\circ}, y_{\circ}\right)=y_{\circ} e^{C t}+\int_{0}^{t} e^{C(t-s)}\left[D(s) y\left(s, x_{\circ}, y_{\circ}\right)+g\left(s, x\left(s, x_{\circ}, y_{\circ}\right), y\left(s, x_{\circ}, y_{\circ}\right)\right)-\right. \\
\left.-\frac{C}{e^{C T}-E} \int_{0}^{T} e^{C(T-s)}\left[D(s) y\left(s, x_{\circ}, y_{\circ}\right)+g\left(s, x\left(s, x_{\circ}, y_{\circ}\right), y\left(s, x_{\circ}, y_{\circ}\right)\right)\right] d s\right] d s
\end{gathered}
$$

which are a unique solution of the system (1) provided that:

$$
\left(\begin{array}{c}
\left\|x^{\circ}\left(t, x_{\circ}, y_{\circ}\right)-x_{m}\left(t, x_{\circ}, y_{\circ}\right)\right\| \\
\left\|y^{\circ}\left(t, x_{\circ}, y_{\circ}\right)-y_{m}\left(t, x_{\circ}, y_{\circ}\right)\right\|
\end{array}\right) \leq \Omega_{\circ}^{m}\left(E-\Omega_{\circ}\right)^{-1} Z_{\circ}
$$

for all $m \geq 1$ and $t \in R^{\prime}$.

where $Z_{\circ}=\left(\begin{array}{l}\frac{T}{2} N+Q C_{1} \\ \frac{T}{2} N^{*}+R \delta_{1}\end{array}\right), \quad$ E the identity matrix

\section{Proof :-}

Each of the following sequences which are defined by (1.4) and (1.5) and has the form

$$
\begin{aligned}
& x_{1}\left(t, x_{\circ}, y_{\circ}\right), x_{2}\left(t, x_{\circ}, y_{\circ}\right), \ldots \ldots, x_{m}\left(t, x_{\circ}, y_{\circ}\right), \ldots \ldots . . \\
& y_{1}\left(t, x_{\circ}, y_{\circ}\right), y_{2}\left(t, x_{\circ}, y_{\circ}\right), \ldots \ldots, y_{m}\left(t, x_{\circ}, y_{\circ}\right), \ldots \ldots . .
\end{aligned}
$$

are defined, continuous in the domain (2) and periodic in t of period $\mathrm{T}$.

By lemma 1 and from (1.4) when $\mathrm{m}=0$ we obtain

$\left\|x_{1}\left(t, x_{\circ}, y_{\circ}\right)-x_{\circ}\left(t, x_{\circ}, y_{\circ}\right)\right\| \leq \beta_{1}(t) N+Q C_{1} \leq \frac{T}{2} N+Q C_{1}$

Thus $x_{1}\left(t, x_{\circ}, y_{\circ}\right) \in D$ for all $x_{\circ} \in D_{f}, y_{\circ} \in D_{g}$ also from the relation (19) and by lemma 1 when $m=0$ we obtain

$$
\left\|y_{1}\left(t, x_{\circ}, y_{\circ}\right)-y_{\circ}\left(t, x_{\circ}, y_{\circ}\right)\right\| \leq \beta_{2}(t) N^{*}+R \delta_{1} \leq \frac{T}{2} N^{*}+R \delta_{1}
$$




\section{R.N.Butris \& Merna Adel Aziz}

and this gives $y_{1}\left(t, x_{\circ}, y_{\circ}\right) \in D_{1}$ for all $x_{\circ} \in D_{f}, y_{\circ} \in D_{g}$

By using the mathematical induction we can prove the truth of the following inequalities for $m \geq 1$

$\left.\begin{array}{c}\left\|x_{m}\left(t, x_{\circ}, y_{\circ}\right)-x_{\circ}\left(t, x_{\circ}, y_{\circ}\right)\right\| \leq \frac{T}{2} N+Q C_{1} \\ \left\|y_{m}\left(t, x_{\circ}, y_{\circ}\right)-y_{\circ}\left(t, x_{\circ}, y_{\circ}\right)\right\| \leq \frac{T}{2} N^{*}+R \delta_{1}\end{array}\right\}$

that is $x_{m}\left(t, x_{\circ}, y_{\circ}\right) \in D, y_{m}\left(t, x_{\circ}, y_{\circ}\right) \in D_{1}$ for all $x_{\circ} \in D_{f}, y_{\circ} \in D_{g}$

Now we prove that the sequences of functions $\left\{x_{m}\left(t, x_{\circ}, y_{\circ}\right)\right\}_{m=0}^{\infty}$ and $\left\{y_{m}\left(t, x_{\circ}, y_{\circ}\right)\right\}_{m=0}^{\infty}$ are uniformly convergent in the domain (1.6) and also each the sequences of functions are periodic and continuous in the same domain.

Now when $m=1$ in (1.4) and by using lemma 1 we find that

$$
\begin{aligned}
\left\|x_{2}\left(t, x_{\circ}, y_{\circ}\right)-x_{1}\left(t, x_{\circ}, y_{\circ}\right)\right\| \leq\left[\beta_{1}(t) Q H+\right. & \left.Q C_{2}\right]\left\|x_{1}\left(t, x_{\circ}, y_{\circ}\right)-x_{\circ}\left(t, x_{\circ}, y_{\circ}\right)\right\|+ \\
& +Q C_{3}\left\|y_{1}\left(t, x_{\circ}, y_{\circ}\right)-y_{\circ}\left(t, x_{\circ}, y_{\circ}\right)\right\|
\end{aligned}
$$

Also when $\mathrm{m}=1$ in (1.4) and by using lemma 1 we find that

$$
\begin{aligned}
\left\|y_{2}\left(t, x_{\circ}, y_{\circ}\right)-y_{1}\left(t, x_{\circ}, y_{\circ}\right)\right\| & \leq R \delta_{2}\left\|x_{1}\left(t, x_{\circ}, y_{\circ}\right)-x_{\circ}\left(t, x_{\circ}, y_{\circ}\right)\right\|+ \\
+ & {\left[\beta_{2}(t) R J+R \delta_{3}\right]\left\|y_{1}\left(t, x_{\circ}, y_{\circ}\right)-y_{\circ}\left(t, x_{\circ}, y_{\circ}\right)\right\| }
\end{aligned}
$$

So by using the mathematical induction we can prove the truth of the following inequalities:

$$
\begin{aligned}
\left\|x_{m+1}\left(t, x_{\circ}, y_{\circ}\right)-x_{m}\left(t, x_{\circ}, y_{\circ}\right)\right\| & \leq\left[\beta_{1}(t) Q H+Q C_{2}\right]\left\|x_{m}\left(t, x_{\circ}, y_{\circ}\right)-x_{m-1}\left(t, x_{\circ}, y_{\circ}\right)\right\|+ \\
+ & Q C_{3}\left\|y_{m}\left(t, x_{\circ}, y_{\circ}\right)-y_{m-1}\left(t, x_{\circ}, y_{\circ}\right)\right\| \\
\left\|y_{m+1}\left(t, x_{\circ}, y_{\circ}\right)-y_{m}\left(t, x_{\circ}, y_{\circ}\right)\right\| & \leq R \delta_{2}\left\|x_{m}\left(t, x_{\circ}, y_{\circ}\right)-x_{m-1}\left(t, x_{\circ}, y_{\circ}\right)\right\|+ \\
+ & {\left[\beta_{2}(t) R J+R \delta_{3}\right]\left\|y_{m}\left(t, x_{\circ}, y_{\circ}\right)-y_{m-1}\left(t, x_{\circ}, y_{\circ}\right)\right\| }
\end{aligned}
$$

we rewrite (1.13) and (1.14) in vector form as

$$
Z_{m+1}(t) \leq \Omega(t) Z_{m}(t)
$$

where

$$
\begin{aligned}
& Z_{m+1}(t)=\left(\begin{array}{cc}
\left\|x_{m+1}\left(t, x_{\circ}, y_{\circ}\right)-x_{m}\left(t, x_{\circ}, y_{\circ}\right)\right\| \\
\left\|y_{m+1}\left(t, x_{\circ}, y_{\circ}\right)-y_{m}\left(t, x_{\circ}, y_{\circ}\right)\right\|
\end{array}\right) \\
& \Omega(t)=\left[\begin{array}{lc}
\beta_{1}(t) Q H+Q C_{2} & Q C_{3} \\
R \delta_{2} & \beta_{2}(t) R J+R \delta_{3}
\end{array}\right]
\end{aligned}
$$




$$
Z_{m}(t)=\left(\begin{array}{c}
\left\|x_{m}\left(t, x_{\circ}, y_{\circ}\right)-x_{m-1}\left(t, x_{\circ}, y_{\circ}\right)\right\| \\
\left\|y_{m}\left(t, x_{\circ}, y_{\circ}\right)-y_{m-1}\left(t, x_{\circ}, y_{\circ}\right)\right\|
\end{array}\right)
$$

Now we take the maximum value for the two sides of the inequality (1.15) with

$\beta_{2}(t) \leq \frac{T}{2}, \beta_{1}(t) \leq \frac{T}{2}, 0 \leq t \leq T$

we find that

$$
Z_{m+1} \leq \Omega_{\circ} Z_{m}
$$

where $\Omega_{\circ}=\max _{t \in[0, T]} \Omega(t)$,

$$
\Omega_{\circ}=\left[\begin{array}{lr}
\frac{T}{2} Q H+Q C_{2} & Q C_{3} \\
R \delta_{2} & \frac{T}{2} R J+R \delta_{3}
\end{array}\right]
$$

and by (1.16) we have

$$
Z_{m+1} \leq \Omega_{\circ}^{m} Z_{\circ} \text {, }
$$

and then

$\sum_{i=1}^{m} Z_{i} \leq \sum_{i=1}^{m} \Omega_{\circ}^{i-1} Z_{\circ}$,

since the matrix $\Omega$ 。 has the greatest eigen value given by (12), this insures that the sequence (1.17) is uniformly convergent, that is

$$
\lim _{m \rightarrow \infty} \sum_{i=1}^{m} \Omega_{\circ}^{i-1} Z_{\circ}=\sum_{i=1}^{\infty} \Omega_{\circ}^{i-1} Z_{\circ}=\left(E-\Omega_{\circ}\right)^{-1} Z_{\circ}
$$

and then the relation (1.18) as certain on the convergence of sequences of the functions $\left[x_{m}\left(t, x_{\circ}, y_{\circ}\right), y_{m}\left(t, x_{\circ}, y_{\circ}\right)\right]$ on the domain (1.6).

Let

$$
\left.\begin{array}{l}
\lim _{m \rightarrow \infty} x_{m}\left(t, x_{\circ}, y_{\circ}\right)=x^{\circ}\left(t, x_{\circ}, y_{\circ}\right) \\
\lim _{m \rightarrow \infty} y_{m}\left(t, x_{\circ}, y_{\circ}\right)=y^{\circ}\left(t, x_{\circ}, y_{\circ}\right)
\end{array}\right\}
$$

since each of the sequences of the functions (1.4),(1.5) are continuous and periodic in $t$ of period $\mathrm{T}$ then the limiting of (1.4) and (1.5) are continuous and periodic in t of period $\mathrm{T}$ and $x^{\circ}\left(t, x_{\circ}, y_{\circ}\right)=x\left(t, x_{\circ}, y_{\circ}\right), y^{\circ}\left(t, x_{\circ}, y_{\circ}\right)=y\left(t, x_{\circ}, y_{\circ}\right)$ 
Thus by lemma 1 and the relation (1.19), the inequality (1.9) is satisfied for $\mathrm{m}=0,1,2, \ldots \ldots$.

Now we prove that $x\left(t, x_{\circ}, y_{\circ}\right), y\left(t, x_{\circ}, y_{\circ}\right)$ are unique solution for the system (1) by contradiction .

We assume that there is two different solutions $x\left(t, x_{\circ}, y_{\circ}\right), y\left(t, x_{\circ}, y_{\circ}\right)$ and $\hat{x}\left(t, x_{\circ}, y_{\circ}\right), \hat{y}\left(t, x_{\circ}, y_{\circ}\right)$ for the system (1) which are defined, continuous and periodic in $\mathrm{t}$ of period $\mathrm{T}$.

That is:

$$
\begin{gathered}
\hat{x}\left(t, x_{\circ}, y_{\circ}\right)=x_{\circ} e^{A t}+\int_{0}^{t} e^{A(t-s)}\left[B(s) \hat{x}\left(s, x_{\circ}, y_{\circ}\right)+f\left(s, \hat{x}\left(s, x_{\circ}, y_{\circ}\right), \hat{y}\left(s, x_{\circ}, y_{\circ}\right)\right)-\right. \\
\left.-\frac{A}{e^{A T}-E} \int_{0}^{T} e^{A(T-s)}\left[B(s) \hat{x}\left(s, x_{\circ}, y_{\circ}\right)+f\left(s, \hat{x}\left(s, x_{\circ}, y_{\circ}\right), \hat{y}\left(s, x_{\circ}, y_{\circ}\right)\right)\right] d s\right] d s \ldots \\
\hat{y}\left(t, x_{\circ}, y_{\circ}\right)=y_{\circ} e^{C t}+\int_{0}^{t} e^{C(t-s)}\left[D(s) \hat{y}\left(s, x_{\circ}, y_{\circ}\right)+g\left(s, \hat{x}\left(s, x_{\circ}, y_{\circ}\right), \hat{y}\left(s, x_{\circ}, y_{\circ}\right)\right)-\right. \\
\left.-\frac{C}{e^{C T}-E} \int_{0}^{T} e^{C(T-s)}\left[D(s) \hat{y}\left(s, x_{\circ}, y_{\circ}\right)+g\left(s, \hat{x}\left(s, x_{\circ}, y_{\circ}\right), \hat{y}\left(s, x_{\circ}, y_{\circ}\right)\right)\right] d s\right] d s
\end{gathered}
$$
have

Now from the integral equations (1.7), (1.8) and (1.20), (1.21) we

$$
\begin{aligned}
\left\|x\left(t, x_{\circ}, y_{\circ}\right)-\hat{x}\left(t, x_{\circ}, y_{\circ}\right)\right\| \leq & {\left[\frac{T}{2} Q H+Q C_{2}\right]\left\|x\left(t, x_{\circ}, y_{\circ}\right)-\hat{x}\left(t, x_{\circ}, y_{\circ}\right)\right\|+} \\
& +Q C_{3} \| y\left(t, x_{\circ}, y_{\circ}\right)-\hat{y}\left(t, x_{\circ}, y_{\circ} \|\right.
\end{aligned}
$$

also

$$
\begin{aligned}
\left\|y\left(t, x_{\circ}, y_{\circ}\right)-\hat{y}\left(t, x_{\circ}, y_{\circ}\right)\right\| \leq R \delta_{2}\left\|x\left(t, x_{\circ}, y_{\circ}\right)-\hat{x}\left(t, x_{\circ}, y_{\circ}\right)\right\|+ \\
+\left[\frac{T}{2} R J+R \delta_{3}\right]\left\|y\left(t, x_{\circ}, y_{\circ}\right)-\hat{y}\left(t, x_{\circ}, y_{\circ}\right)\right\|
\end{aligned}
$$

From (1.22),(1.23) we find that

$$
\left(\begin{array}{l}
\left\|x\left(t, x_{\circ}, y_{\circ}\right)-\hat{x}\left(t, x_{\circ}, y_{\circ}\right)\right\| \\
\left\|y\left(t, x_{\circ}, y_{\circ}\right)-\hat{y}\left(t, x_{\circ}, y_{\circ}\right)\right\|
\end{array}\right) \leq \Omega_{\circ}\left(\begin{array}{l}
\left\|x\left(t, x_{\circ}, y_{\circ}\right)-\hat{x}\left(t, x_{\circ}, y_{\circ}\right)\right\| \\
\left\|y\left(t, x_{\circ}, y_{\circ}\right)-\hat{y}\left(t, x_{\circ}, y_{\circ}\right)\right\|
\end{array}\right)
$$

by continuation this process we have

$$
\left(\begin{array}{l}
\left\|x\left(t, x_{\circ}, y_{\circ}\right)-\hat{x}\left(t, x_{\circ}, y_{\circ}\right)\right\| \\
\left\|y\left(t, x_{\circ}, y_{\circ}\right)-\hat{y}\left(t, x_{\circ}, y_{\circ}\right)\right\|
\end{array}\right) \leq \Omega_{\circ}^{m}\left(\begin{array}{l}
\left\|x\left(t, x_{\circ}, y_{\circ}\right)-\hat{x}\left(t, x_{\circ}, y_{\circ}\right)\right\| \\
\left\|y\left(t, x_{\circ}, y_{\circ}\right)-\hat{y}\left(t, x_{\circ}, y_{\circ}\right)\right\|
\end{array}\right)
$$

but from the condition (12) we get $\Omega_{\circ}^{m} \rightarrow 0$ when $m \rightarrow \infty$ and thus we have 
$x\left(t, x_{\circ}, y_{\circ}\right)=\hat{x}\left(t, x_{\circ}, y_{\circ}\right), y\left(t, x_{\circ}, y_{\circ}\right)=\hat{y}\left(t, x_{\circ}, y_{\circ}\right)$ that is each of $x\left(t, x_{\circ}, y_{\circ}\right), y\left(t, x_{\circ}, y_{\circ}\right)$ are unique solution for the system (1)

\section{Section Two : The existence of the periodic solution}

\section{for the system (1)}

The problem of the existence of the periodic solution for the system (1) is uniquely connected with the existence of zeros the functions $\Delta_{1}^{*}=\Delta_{1}^{*}\left(x_{\circ}, y_{\circ}\right), \Delta_{2}^{*}=\Delta_{2}^{*}\left(x_{\circ}, y_{\circ}\right)$ where:

$$
\begin{aligned}
\Delta_{1}\left(x_{\circ}, y_{\circ}\right)=\left(\frac{A}{e^{A T}-E}\right)_{0}^{T} \int_{0}^{T} e^{A(T-t)}\left[B(t) x^{\circ}\left(t, x_{\circ}, y_{\circ}\right)+\right. \\
\left.+f\left(t, x^{\circ}\left(t, x_{\circ}, y_{\circ}\right), y^{\circ}\left(t, x_{\circ}, y_{\circ}\right)\right)\right] d t
\end{aligned}
$$

and

$$
\begin{aligned}
\Delta_{2}\left(x_{\circ}, y_{\circ}\right)=\left(\frac{C}{e^{C T}-E}\right)_{0}^{T} \int_{0}^{C(T-t)}\left[D(t) y^{\circ}\left(t, x_{\circ}, y_{\circ}\right)+\right. \\
\left.+g\left(t, x^{\circ}\left(t, x_{\circ}, y_{\circ}\right), y^{\circ}\left(t, x_{\circ}, y_{\circ}\right)\right)\right] d t
\end{aligned}
$$

Since these functions is approximately determined from the sequence of functions

$$
\begin{aligned}
\Delta_{1 m}\left(x_{\circ}, y_{\circ}\right)=\left(\frac{A}{e^{A T}-E}\right)_{0}^{T} \int_{0}^{A(T-t)}\left[B(t) x_{m}\left(t, x_{\circ}, y_{\circ}\right)+\right. \\
\left.+f\left(t, x_{m}\left(t, x_{\circ}, y_{\circ}\right), y_{m}\left(t, x_{\circ}, y_{\circ}\right)\right)\right] d t
\end{aligned}
$$

and

$$
\begin{aligned}
\Delta_{2 m}\left(x_{\circ}, y_{\circ}\right)=\left(\frac{C}{e^{C T}-E}\right)_{0}^{T} \int_{0}^{C(T-t)}\left[D(t) y_{m}\left(t, x_{\circ}, y_{\circ}\right)+\right. \\
\left.+g\left(t, x_{m}\left(t, x_{\circ}, y_{\circ}\right), y_{m}\left(t, x_{\circ}, y_{\circ}\right)\right)\right] d t
\end{aligned}
$$

where $\mathrm{m}=0,1,2, \ldots \ldots$.

\section{Theorem 2:-}

Under the assumptions of theorem 1 we have the following inequalities:

$$
\begin{aligned}
\left\|\Delta_{1}\left(x_{\circ}, y_{\circ}\right)-\Delta_{1 m}\left(x_{\circ}, y_{\circ}\right)\right\| \leq & \left\langle\left(\begin{array}{lr}
\left(N_{1}^{*} Q\left(T H+C_{2}\right)\right. & \left.\left.N_{1}^{*} Q C_{3}\right), \Omega_{\circ}^{m}\left(E-\Omega_{\circ}\right)^{-1} Z_{\circ}\right\rangle \\
& =\rho_{m}^{*} \\
\left\|\Delta_{2}\left(x_{\circ}, y_{\circ}\right)-\Delta_{2 m}\left(x_{\circ}, y_{\circ}\right)\right\| \leq & \left\langle\begin{array}{ll}
\left(N_{2}^{*} R \delta_{2}\right. & \left.N_{2}^{*} R\left(T J+\delta_{3}\right)\right), \Omega_{\circ}^{m}\left(E-\Omega_{\circ}\right)^{-1} Z_{\circ}
\end{array}\right\rangle \\
& =\gamma_{m}^{*}
\end{array}\right.\right.
\end{aligned}
$$


Where $N_{1}^{*}=\frac{\|A\|}{e^{\|A\| T}-\|E\|}, N_{2}^{*}=\frac{\|C\|}{e^{\|C\| T}-\|E\|}, \rho_{m}^{*}, \gamma_{m}^{*}$ are positive

numbers, with $m \geq 0, x_{\circ} \in D_{f}, y_{\circ} \in D_{g}$

and $\langle$.$\rangle denote to the scalar product in the Euclidean space R^{n}$.

\section{Proof :-}

By equations (2.1), (2.3) and the condition (1.9) we have $\left\|\Delta_{1}\left(x_{\circ}, y_{\circ}\right)-\Delta_{1 m}\left(x_{\circ}, y_{\circ}\right)\right\| \leq$

$$
\begin{aligned}
& \leq\left(\frac{\|A\|}{e^{\|A\| T}-\|E\|}\right)\left[Q\left(T H+C_{2}\right)\left\|x^{\circ}\left(t, x_{\circ}, y_{\circ}\right)-x_{m}\left(t, x_{\circ}, y_{\circ}\right)\right\|+\right. \\
& \left.\quad+Q C_{3}\left\|y^{\circ}\left(t, x_{\circ}, y_{\circ}\right)-y_{m}\left(t, x_{\circ}, y_{\circ}\right)\right\|\right] \\
& \leq\left\langle\left(N_{1}^{*} Q\left(T H+C_{2}\right) \quad N_{1}^{*} Q C_{3}\right), \Omega_{\circ}^{m}\left(E-\Omega_{\circ}\right)^{-1} Z_{\circ}\right\rangle=\rho_{m}^{*}
\end{aligned}
$$

also by using equations (2.2), (2.4) we have

$$
\begin{aligned}
& \left\|\Delta_{2}\left(x_{\circ}, y_{\circ}\right)-\Delta_{2 m}\left(x_{\circ}, y_{\circ}\right)\right\| \leq \\
\leq & \left(\frac{\|C\| T}{e^{\|C\| T}-\|E\|}\right)\left[R\left(T J+\delta_{3}\right)\left\|y^{\circ}\left(t, x_{\circ}, y_{\circ}\right)-y_{m}\left(t, x_{\circ}, y_{\circ}\right)\right\|+\right. \\
& \left.\quad+R \delta_{2}\left\|x^{\circ}\left(t, x_{\circ}, y_{\circ}\right)-x_{m}\left(t, x_{\circ}, y_{\circ}\right)\right\|\right] \\
& \leq\left\langle\left(N_{2}^{*} R \delta_{2} \quad N_{2}^{*} R\left(T J+\delta_{3}\right)\right), \Omega_{\circ}^{m}\left(E-\Omega_{\circ}\right)^{-1} Z_{\circ}\right\rangle=\gamma_{m}^{*}
\end{aligned}
$$

\section{Remark 1 :-}

When $R^{n}=R^{1}$, i.e when $\mathrm{x}$ and y are a scalar points, thus we have

\section{Theorem 3:-}

Let the functions $f(t, x, y)$ and $g(t, x, y)$ of the system (1) are defined on the intervals [a, b] ,[c, d] in $\mathrm{R}^{1}$ and for any integer $m \geq 1$ the functions (2.3), (2.4) satisfies the inequalities: 


$$
\begin{aligned}
& \min \Delta_{1 m}\left(x_{\circ}, y_{\circ}\right) \leq-\rho_{m}^{*} \\
& a+h \leq x_{\circ} \leq b-h \\
& c+h^{*} \leq y_{\circ} \leq d-h^{*} \\
& \max \Delta_{1 m}\left(x_{\circ}, y_{\circ}\right) \geq \rho_{m}^{*} \\
& a+h \leq x_{\circ} \leq b-h \\
& c+h^{*} \leq y_{\circ} \leq d-h^{*} \\
& \min \Delta_{2 m}\left(x_{\circ}, y_{\circ}\right) \leq-\gamma_{m}^{*} \\
& a+h \leq x_{\circ} \leq b-h \\
& c+h^{*} \leq y_{\circ} \leq d-h^{*} \\
& \max \Delta_{2 m}\left(x_{\circ}, y_{\circ}\right) \geq \gamma_{m}^{*} \\
& a+h \leq x_{\circ} \leq b-h \\
& c+h^{*} \leq y_{\circ} \leq d-h^{*}
\end{aligned}
$$

For all

$$
\begin{gathered}
N_{1}^{*}=\frac{\|A\|}{e^{\|A\| T}-\|E\|}, \rho_{m}^{*}=\left\langle\left(N_{1}^{*} Q\left(T H+C_{2}\right) \quad N_{1}^{*} Q C_{3}\right), \Omega_{\circ}^{m}\left(E-\Omega_{\circ}\right)^{-1} Z_{\circ}\right\rangle, m \geq 0 \\
N_{2}^{*}=\frac{\|C\|}{e^{\|C\| T}-\|E\|}, \gamma_{m}^{*}=\left\langle\left(N_{2}^{*} R \delta_{2} \quad N_{2}^{*} R\left(T J+\delta_{3}\right)\right), \Omega_{\circ}^{m}\left(E-\Omega_{\circ}\right)^{-1} Z \circ\right\rangle, m \geq 0 \\
h^{*}=\frac{T}{2} N^{*}+R \delta_{1}, h=\frac{T}{2} N+Q C_{1}, \quad N^{*}=R^{2} J \sigma_{\circ}, N=Q^{2} H \delta_{\circ}
\end{gathered}
$$

then the system $x=x\left(t, x_{\circ}, y_{\circ}\right), y=y\left(t, x_{\circ}, y_{\circ}\right)$

(1) have periodic solutions for $x_{\circ} \in[a+h, b-h]$ and $y_{\circ} \in\left[c+h^{*}, d-h^{*}\right]$.

\section{Proof :-}

Let $x_{1}, x_{2}$ be any two points in the interval $[a+h, b-h]$ and $y_{2}, y_{1}$ be any two points in the interval $\left[c+h^{*}, d-h^{*}\right]$ such that 


$$
\begin{aligned}
& \text { R.N.Butris \& Merna Adel Aziz } \\
& \Delta_{1 m}\left(x_{1}, y_{1}\right)=\min \Delta_{1 m}\left(x_{\circ}, y_{\circ}\right) \\
& a+h \leq x_{\circ} \leq b-h \\
& c+h^{*} \leq y_{\circ} \leq d-h^{*} \\
& \Delta_{1 m}\left(x_{2}, y_{2}\right)=\max \Delta_{1 m}\left(x_{\circ}, y_{\circ}\right) \\
& a+h \leq x_{\circ} \leq b-h \\
& c+h^{*} \leq y_{\circ} \leq d-h^{*} \\
& \Delta_{2 m}\left(x_{1}, y_{1}\right)=\min \Delta_{2 m}\left(x_{\circ}, y_{\circ}\right) \\
& a+h \leq x_{\circ} \leq b-h \\
& c+h^{*} \leq y_{\circ} \leq d-h^{*} \\
& \Delta_{2 m}\left(x_{2}, y_{2}\right)=\max \Delta_{2 m}\left(x_{\circ}, y_{\circ}\right) \\
& a+h \leq x_{\circ} \leq b-h \\
& c+h^{*} \leq y_{\circ} \leq d-h^{*} \\
& \left.\begin{array}{l}
\Delta_{1}\left(x_{1}, y_{1}\right)=\Delta_{1 m}\left(x_{1}, y_{1}\right)+\left(\Delta_{1}\left(x_{1}, y_{1}\right)-\Delta_{1 m}\left(x_{1}, y_{1}\right)\right)<0 \\
\Delta_{1}\left(x_{2}, y_{2}\right)=\Delta_{1 m}\left(x_{2}, y_{2}\right)+\left(\Delta_{1}\left(x_{2}, y_{2}\right)-\Delta_{1 m}\left(x_{2}, y_{2}\right)\right)>0
\end{array}\right\} \\
& \Delta_{2}\left(x_{1}, y_{1}\right)=\Delta_{2 m}\left(x_{1}, y_{1}\right)+\left(\Delta_{2}\left(x_{1}, y_{1}\right)-\Delta_{2 m}\left(x_{1}, y_{1}\right)\right)<0 \\
& \left.\Delta_{2}\left(x_{2}, y_{2}\right)=\Delta_{2 m}\left(x_{2}, y_{2}\right)+\left(\Delta_{2}\left(x_{2}, y_{2}\right)-\Delta_{2 m}\left(x_{2}, y_{2}\right)\right)>0\right\}
\end{aligned}
$$

and from the continuity of the functions $\Delta_{1}\left(x_{\circ}, y_{\circ}\right), \Delta_{2}\left(x_{\circ}, y_{\circ}\right)$ and the inequalities (2.11), (2.12) then there exist an isolated singular point $\left(x^{\circ}, y^{\circ}\right)=\left(x_{\circ}, y_{\circ}\right), \quad x^{\circ} \in\left[x_{1}, x_{2}\right]$ and $y^{\circ} \in\left[y_{1}, y_{2}\right] \quad$ where $\Delta_{1}\left(x_{\circ}, y_{\circ}\right)=0, \Delta_{2}\left(x_{\circ}, y_{\circ}\right)=0$.

That is $x=\left(t, x_{\mathrm{o}}, y_{\mathrm{o}}\right)$ and $y=\left(t, x_{\mathrm{o}}, y_{\mathrm{o}}\right)$ a periodic solutions of the system (1), for $x_{\circ} \in[a+h, b-h], y_{\circ} \in\left[c+h^{*}, d-h^{*}\right]$. 


\section{Theorem 4 :-}

Let

$$
\begin{aligned}
& \Delta_{1}: D_{f} \times D_{g} \rightarrow R^{n}, \\
& \Delta_{1}\left(x_{\circ}, y_{\circ}\right)=\left(\frac{A}{e^{A T}-E}\right)_{0}^{T} \int_{0}^{A(T-t)}\left[B(t) x^{\circ}\left(t, x_{\circ}, y_{\circ}\right)+\right. \\
& \left.+f\left(t, x^{\circ}\left(t, x_{\circ}, y_{\circ}\right), y^{\circ}\left(t, x_{\circ}, y_{\circ}\right)\right)\right] d t
\end{aligned}
$$

and

$$
\begin{aligned}
& \Delta_{2}: D_{f} \times D_{g} \rightarrow R^{n}, \\
& \Delta_{2}\left(x_{\circ}, y_{\circ}\right)=\left(\frac{C}{e^{C T}-E}\right)_{0}^{T} \int_{0}^{C(T-t)}\left[D(t) y^{\circ}\left(t, x_{\circ}, y_{\circ}\right)+\right. \\
& \left.+g\left(t, x^{\circ}\left(t, x_{\circ}, y_{\circ}\right), y^{\circ}\left(t, x_{\circ}, y_{\circ}\right)\right)\right] d t
\end{aligned}
$$

where $x^{\circ}\left(t, x_{\circ}, y_{\circ}\right)$ and $y^{\circ}\left(t, x_{\circ}, y_{\circ}\right)$ are the limiting functions of the periodic sequence (1.4) and (1.5), then the following inequalities

$$
\left\|\Delta_{1}\left(x_{\circ}, y_{\circ}\right)\right\| \leq M_{7}
$$

where

$$
\begin{aligned}
& \quad M_{7}=N_{1} Q H\left(\delta_{\circ} Q M_{3}+Q C_{1} M_{3}\right)+N_{1}^{*} Q C_{1}, M_{3}=\left(1-\frac{T}{2} Q H\right)^{-1} \\
& \left\|\Delta_{2}\left(x_{\circ}, y_{\circ}\right)\right\| \leq M_{8}
\end{aligned}
$$

and $\quad M_{8}=N_{2} R J\left(\sigma_{\circ} R M_{5}+R \delta_{1} M_{5}\right)+N_{2}^{*} R \delta_{1}, M_{5}=\left(1-\frac{T}{2} R J\right)^{-1}$ $\left\|\Delta_{1}\left(x_{\circ}^{1}, y_{\circ}^{1}\right)-\Delta_{1}\left(x_{\circ}^{2}, y_{\circ}^{2}\right)\right\| \leq\left[N_{1}^{*} E_{5} W_{1} W_{2}\left(1-E_{4}\right)+N_{1}^{*} E_{2} E_{3} W_{1} W_{2}\right]\left\|x_{\circ}^{1}-x_{\circ}^{2}\right\| Q+$ $+\left[N_{1}^{*} E_{2} E_{5} W_{1} W_{2}+N_{1}^{*} E_{2}\left(1+E_{2} E_{3} W_{1} W_{2}\right)\left(1-E_{4}\right)^{-1}\right]\left\|y_{\circ}^{1}-y_{\circ}^{2}\right\| R$

$\left\|\Delta_{2}\left(x_{\circ}^{1}, y_{\circ}^{1}\right)-\Delta_{2}\left(x_{\circ}^{2}, y_{\circ}^{2}\right)\right\| \leq\left[N_{2}^{*} E_{3} W_{1} W_{2}\left(1-E_{4}\right)+N_{2}^{*} E_{3} E_{4} W_{1} W_{2}\right]\left\|x_{\circ}^{1}-x_{\circ}^{2}\right\| Q+$ $+\left[N_{2}^{*} E_{2} E_{3} W_{1} W_{2}+N_{2}^{*} E_{4}\left(1+E_{2} E_{3} W_{1} W_{2}\right)\left(1-E_{4}\right)^{-1}\right]\left\|y_{\circ}^{1}-y_{\circ}^{2}\right\| R$

satisfies for $x_{\circ}, x_{\circ}^{1}, x_{\circ}^{2} \in D_{f}, y_{\circ}, y_{\circ}^{1}, y_{\circ}^{2} \in D_{g}$

Where

$$
\begin{aligned}
& N_{1}=\frac{\|A\| T}{e^{\|A\| T}-\|E\|}, N_{2}=\frac{\|C\| T}{e^{\|C\| T}-\|E\|}, N_{1}^{*}=\frac{\|A\|}{e^{\|A\| T}-\|E\|}, N_{2}^{*}=\frac{\|C\|}{e^{\|C\| T}-\|E\|} \\
& E_{1=}\left[\frac{T}{2} Q H+Q C_{2}\right], E_{2}=Q C_{3}, E_{3}=R \delta_{2}, E_{4}=\left[\frac{T}{2} R J+R \delta_{3}\right], E_{5}=Q\left(T H+C_{2}\right) \\
& W_{1}=\left[\left(1-E_{1}\right)\left(1-E_{4}\right)\right]^{-1}, W_{2}=\left(1-E_{2} E_{3} W_{1}\right)^{-1}
\end{aligned}
$$




\section{R.N.Butris \& Merna Adel Aziz}

\section{Proof :-}

From the properties of the functions $x^{\circ}\left(t, x_{\circ}, y_{\circ}\right), y^{\circ}\left(t, x_{\circ}, y_{\circ}\right)$ that theorem 1 then each of the functions $\Delta_{1}=\Delta_{1}\left(x_{\circ}, y_{\circ}\right), \Delta_{2}=\Delta_{2}\left(x_{\circ}, y_{\circ}\right)$, $x_{\circ} \in D_{f}, y_{\circ} \in D_{g}$ continuous and bounded by non negative constants $M_{7}, M_{8}$, and from the relation (2.13) we find that:

$\left\|\Delta_{1}\left(x_{\circ}, y_{\circ}\right)\right\| \leq \frac{\|A\| T}{e^{\|A\| T}-\|E\|} Q H\left\|x^{\circ}\left(t, x_{\circ}, y_{\circ}\right)\right\|+\frac{\|A\|}{e^{|A A| T}-\|E\|} Q C_{1}$

since that $x^{\circ}\left(t, x_{\circ}, y_{\circ}\right)$ satisfy the integral equation (1.7) and by using lemma1 we have:

$$
\left\|x^{\circ}\left(t, x_{\circ}, y_{\circ}\right)\right\| \leq \delta_{\circ} Q M_{3}+Q C_{1} M_{3}
$$

and

$$
\left\|\Delta_{2}\left(x_{\circ}, y_{\circ}\right)\right\| \leq \frac{\|C\| T}{e^{\|C\| T}-\|E\|} R J\left\|y^{\circ}\left(t, x_{\circ}, y_{\circ}\right)\right\|+\frac{\|C\|}{e^{\|C\| T}-\|E\|} R \delta_{1},
$$

since that $y^{\circ}\left(t, x_{\circ}, y_{\circ}\right)$ satisfy the integral equation (1.8) and by using lemma1 we find that

$$
\left\|y^{\circ}\left(t, x_{\circ}, y_{\circ}\right)\right\| \leq \sigma_{\circ} R M_{5}+R \delta_{1} M_{5}
$$

and from the relation (2.13) we get:

$$
\begin{aligned}
\left\|\Delta_{1}\left(x_{\circ}^{1}, y_{\circ}^{1}\right)-\Delta_{1}\left(x_{\circ}^{2}, y_{\circ}^{2}\right)\right\| & \leq N_{1}^{*} E_{5}\left\|x^{\circ}\left(t, x_{\circ}^{1}, y_{\circ}^{1}\right)-x^{\circ}\left(t, x_{\circ}^{2}, y_{\circ}^{2}\right)\right\|+ \\
& +N_{1}^{*} E_{2}\left\|y^{\circ}\left(t, x_{\circ}^{1}, y_{\circ}^{1}\right)-y^{\circ}\left(t, x_{\circ}^{2}, y_{\circ}^{2}\right)\right\|
\end{aligned}
$$

where

$$
\begin{aligned}
& x\left(t, x_{\circ}^{k}, y_{\circ}^{k}\right)=x_{\circ}^{k} e^{A t}+\int_{0}^{t} e^{A(t-s)}\left[B(s) x\left(s, x_{\circ}^{k}, y_{\circ}^{k}\right)+f\left(s, x\left(s, x_{\circ}^{k}, y_{\circ}^{k}\right), y\left(s, x_{\circ}^{k}, y_{\circ}^{k}\right)\right)-\right. \\
& \left.-\frac{A}{e^{A T}-E} \int_{0}^{T} e^{A(T-s)}\left[B(s) x\left(s, x_{\circ}^{k}, y_{\circ}^{k}\right)+f\left(s, x\left(s, x_{\circ}^{k}, y_{\circ}^{k}\right), y\left(s, x_{\circ}^{k}, y_{\circ}^{k}\right)\right)\right] d s\right] d s \ldots \ldots . .(2.24) \\
& y\left(t, x_{\circ}^{k}, y_{\circ}^{k}\right)=y_{\circ}^{k} e^{C t}+\int_{0}^{t} e^{C(t-s)}\left[D(s) y\left(s, x_{\circ}^{k}, y_{\circ}^{k}\right)+g\left(s, x\left(s, x_{\circ}^{k}, y_{\circ}^{k}\right), y\left(s, x_{\circ}^{k}, y_{\circ}^{k}\right)\right)-\right. \\
& \left.-\frac{C}{e^{C T}-E} \int_{0}^{T} e^{C(T-s)}\left[D(s) y\left(s, x_{\circ}^{k}, y_{\circ}^{k}\right)+g\left(s, x\left(s, x_{\circ}^{k}, y_{\circ}^{k}\right), y\left(s, x_{\circ}^{k}, y_{\circ}^{k}\right)\right)\right] d s\right] d s \ldots \ldots .(2.25)
\end{aligned}
$$


where $\mathrm{k}=1,2$.

since $x^{\circ}\left(t, x_{\circ}, y_{\circ}\right), y^{\circ}\left(t, x_{\circ}, y_{\circ}\right)$ satisfies the two equations (1.7),(1.8) on arrangement.

from the relation (2.24) we find that

$$
\begin{aligned}
\left\|x^{\circ}\left(t, x_{\circ}^{1}, y_{\circ}^{1}\right)-x^{\circ}\left(t, x_{\circ}^{2}, y_{\circ}^{2}\right)\right\| \leq & \left(1-E_{1}\right)^{-1}\left\|x_{\circ}^{1}-x_{\circ}^{2}\right\| Q+ \\
& +E_{2}\left(1-E_{1}\right)^{-1}\left\|y^{\circ}\left(t, x_{\circ}^{1}, y_{\circ}^{1}\right)-y^{\circ}\left(t, x_{\circ}^{2}, y_{\circ}^{2}\right)\right\|
\end{aligned}
$$

also from the relation (2.25) we find that

$$
\begin{aligned}
\left\|y^{\circ}\left(t, x_{\circ}^{1}, y_{\circ}^{1}\right)-y^{\circ}\left(t, x_{\circ}^{2}, y_{\circ}^{2}\right)\right\| \leq & \left(1-E_{1}\right)^{-1}\left\|y_{\circ}^{1}-y_{\circ}^{2}\right\| R+ \\
& +E_{3}\left(1-E_{4}\right)^{-1}\left\|x^{\circ}\left(t, x_{\circ}^{1}, y_{\circ}^{1}\right)-x^{\circ}\left(t, x_{\circ}^{2}, y_{\circ}^{2}\right)\right\|
\end{aligned}
$$

by substitutions the inequality (2.27) in the inequality (2.26) we obtain

$$
\begin{aligned}
\left\|x^{\circ}\left(t, x_{\circ}^{1}, y_{\circ}^{1}\right)-x^{\circ}\left(t, x_{\circ}^{2}, y_{\circ}^{2}\right)\right\| \leq\left(1-E_{1}\right)^{-1} \| & x_{\circ}^{1}-x_{\circ}^{2}\left\|Q+E_{2} W_{1}\right\| y_{\circ}^{1}-y_{\circ}^{2} \| R+ \\
& +E_{2} E_{3} W_{1}\left\|x^{\circ}\left(t, x_{\circ}^{1}, y_{\circ}^{1}\right)-x^{\circ}\left(t, x_{\circ}^{2}, y_{\circ}^{2}\right)\right\|
\end{aligned}
$$

since $W_{1}\left(1-E_{4}\right)=\left(1-E_{1}\right)^{-1}$ then

$$
\left\|x^{\circ}\left(t, x_{\circ}^{1}, y_{\circ}^{1}\right)-x^{\circ}\left(t, x_{\circ}^{2}, y_{\circ}^{2}\right)\right\| \leq W_{1} W_{2}\left(1-E_{4}\right)\left\|x_{\circ}^{1}-x_{\circ}^{2}\right\| Q+E_{2} W_{1} W_{2}\left\|y_{\circ}^{1}-y_{\circ}^{2}\right\| R
$$

by substitutions the inequality (2.28) in the inequality (2.27) we obtain

$$
\begin{aligned}
\left\|y^{\circ}\left(t, x_{\circ}^{1}, y_{\circ}^{1}\right)-y^{\circ}\left(t, x_{\circ}^{2}, y_{\circ}^{2}\right)\right\| \leq & {\left[1+E_{2} E_{3} W_{1} W_{2}\right]\left(1-E_{4}\right)^{-1}\left\|y_{\circ}^{1}-y_{\circ}^{2}\right\| R+} \\
& +E_{3} W_{1} W_{2}\left\|x_{\circ}^{1}-x_{\circ}^{2}\right\| Q
\end{aligned}
$$

by substitutions the two inequalities (2.28),(2.29) in the inequality (2.23) we obtain (2.17).

also by the relation (2.14) we find that

$$
\begin{aligned}
\left\|\Delta_{2}\left(x_{\circ}^{1}, y_{\circ}^{1}\right)-\Delta_{2}\left(x_{\circ}^{2}, y_{\circ}^{2}\right)\right\| & \leq N_{2}^{*} E_{3}\left\|x^{\circ}\left(t, x_{\circ}^{1}, y_{\circ}^{1}\right)-x^{\circ}\left(t, x_{\circ}^{2}, y_{\circ}^{2}\right)\right\|+ \\
& +N_{2}^{*} E_{4}\left\|y^{\circ}\left(t, x_{\circ}^{1}, y_{\circ}^{1}\right)-y^{\circ}\left(t, x_{\circ}^{2}, y_{\circ}^{2}\right)\right\|
\end{aligned}
$$

by substitutions the inequalities (2.28),(2.29) in the inequality (2.30) we obtain (2.18)

\section{Remark 2 :-}

By [4], we conclude that theorem 4 insures the stability of the solution for the system (1) of non linear differential equations since a slight change in the point $\left(x_{\circ}, y_{\circ}\right)$ leads to a slight change in the functions $\Delta_{1}=\Delta_{1}\left(x_{\circ}, y_{\circ}\right), \Delta_{2}=\Delta_{2}\left(x_{\circ}, y_{\circ}\right)$. 


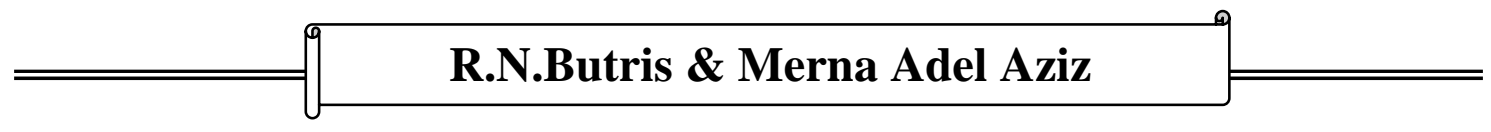

\section{REFERENCES}

1- Butris, R. N. and Saied, EL. M. On periodic solutions for certain systems of integro - differential equations, Iraq, Mosul, J. of Educ. And Sci, Vol.31, p.p. 80-90, (1998).

2- Butris, R. N. and AL -Ameen , M .S. The existence of periodic solutions for nonlinear systems of integro - differential equations, Iraq, Mosul, J. of Educ. And Sci Vol. 34, 37-48, (1999).

3- Martynuk, D. I. Periodic solutions of second - order nonlinear differential equation Ukrain, Math. J. No.4, pp. 125-132, (1967).

4- Mitropolsky, Yu. A. and Martynuk, D. I. Periodic solutions for the oscillations systems with retarded arguments, Ukrain, Kiev, (1979).

5- Naima, D. M. Periodic solutions of non autonomous second - order differential equations boundary value problems - numerical solutions differential equations, nonlinear studies, Vol. 6, Issue.8-14, (1999).

6- Perestyuk, N. A. The periodic solution for nonlinear systems of differential equation, Ukrain, Kiev, univ of kiev, Math. and Meca. J. No. 5, (1971).

7- Samoilenko, A . M. A numerical-analytic methods for investigations of periodic systems of ordinary differential equations I , II , Ukrain, Kiev, $\quad$ Math. J. No.4,5 , p.p. (82-93),(50-59),(1965,1966). 